\title{
Rituximab in severe skin diseases: target, disease, and dose
}

\author{
Daniel D Bennett' \\ Maro Ohanian ${ }^{2}$ \\ Christian T Cable ${ }^{2}$ \\ 'Department of Dermatology, \\ ${ }^{2}$ Department of Internal Medicine, \\ Division of Hematology and Oncology, \\ Scott and White Healthcare, Texas \\ A\&M Health Science Center College \\ of Medicine. Temple, TX, USA
}

\author{
This article was published in the following Dove Press journal: \\ Clinical Pharmacology:Advances and Applications \\ 3 July 2010 \\ Number of times this article has been viewed
}

Correspondence: Christian T Cable Division of Hematology and Oncology, Scott and White Healthcare, 240I S.

3 I st Street, Temple, TX 76508, USA

Tel + I 254724 I 7 I3

Fax: + I 2547244904

Email ccable@swmail.sw.org

\begin{abstract}
New clinical indications for rituximab seem to appear every day. This review will trace the use of this monoclonal antibody from lymphoid malignancy, to classic autoimmune disease, and specifically severe autoimmune skin diseases. The history leading to different dosing schema with associated pharmacokinetic data will be discussed. A case of livedoid vasculopathy (atrophie blanche) responding to rituximab will illustrate how the response to therapy can help to elucidate previously obscure pathophysiology.
\end{abstract}

Keywords: rituximab, livedoid vasculopathy, pharmacokinetics, pemphigus vulgaris, atopic dermatitis, rheumatoid arthritis

\section{Introductory case}

A 49-year-old man diagnosed with livedoid vasculopathy (LV) (atrophie blanche) is referred by dermatology to the hematology clinic for consideration of rituximab therapy. The referring physician had previously treated the patient with systemic corticosteroids as well as multiple steroid sparing immunosuppressive, anticoagulant, and fibrinolytic regimens. Despite compliance, the patient developed corticosteroid-induced diabetes mellitus with lower extremity neuropathy and was unable to work due to painful lower extremity ulcerative lesions requiring opioid analgesia.

The diagnosis of LV had been made by an outside dermatologist after a biopsy of the skin and an evaluation at another tertiary referral center. Upon presentation to our dermatology clinic, he had painful ulcers and white stellate scars involving both lower legs, ankles and dorsal feet consistent with a diagnosis of LV. The patient's ulcers typically healed with tapering courses of prednisone beginning at $1 \mathrm{mg} / \mathrm{kg}$ daily; however, he found that he could rarely go without prednisone for more than two weeks. He was taking colchicine $0.6 \mathrm{mg}$ twice daily as a steroid sparing agent and had previously failed therapy with pentoxyphylline and aspirin.

Prior to and after presentation to our clinic, the patient underwent an extensive laboratory evaluation to assess for autoimmune or procoagulant disorders. Studies that were either negative or within normal limits included: antineutrophil cytoplasmic antibodies, cryoglobulins, rheumatoid factor, lupus anticoagulant screen, anticardiolipin antibodies, prothrombin time, activated partial thromboplastin time, platelet count, hepatitis B and C serologies, homocysteine, erythrocyte sedimentation rate, complement levels, functional protein $\mathrm{C}$ and $\mathrm{S}$, and factor $\mathrm{V}$ Leiden mutation analysis. Antinuclear antibodies were positive at titers of 1:160 and 1:80 on two separate occa- 
sions. An incisional biopsy performed at an outside facility was evaluated by a dermatopathologist and was felt to be consistent with a diagnosis of LV.

Over the course of three years, the patient was treated with multiple agents. Dapsone $50 \mathrm{mg}$ daily provided no benefit; however, clopidogrel bisulfate $75 \mathrm{mg}$ daily combined with colchicine $0.6 \mathrm{mg}$ twice daily initially produced significant improvement with a dramatic reduction in the patient's need for prednisone. Unfortunately, the patient's disease flared-up again after several months. Azathioprine $100 \mathrm{mg}$ daily was added twice although discontinued secondary to elevated transaminases first and transient leukopenia second. Stanazol also failed to produce any improvement. The patient required prednisone as high as $80 \mathrm{mg}$ daily, and his course was complicated by the development of mononeuritis multiplex involving both lower extremities as well as corticosteroidinduced diabetes mellitus.

A single case report describing the successful treatment of this condition with rituximab accompanied the consultation request. ${ }^{1}$ The patient was treated with two cycles of rituximab $1000 \mathrm{mg}$ delivered two weeks apart. Figure 1 demonstrates response to therapy. A recurrence of his disease seven months later responded quickly to further treatment with rituximab.

\section{Livedoid vasculopathy (atrophie blanche)}

LV is characterized by the development of painful purpuric and petechial lesions on the lower extremities. The lesions evolve to "punched-out" ulcers that heal with white, stellate scars. Biopsies of involved skin typically show little inflammation and hyalinized vessels. ${ }^{2}$ Neuropathy in association with LV has been described. ${ }^{3}$ While pathogenesis is obscure, the clinical findings of LV have been associated with both procoagulant and autoimmune diseases. ${ }^{4,2}$ Others have reported clinical LV associated with vasculitis. ${ }^{5}$ The clinical findings have multiple competing and overlapping putative mechanisms, including hypercoagulability, autoimmunity, and idiopathic mechanisms.

Zeni and colleagues reported a single case of LV successfully treated with rituximab $1000 \mathrm{mg}$ two weeks apart. ${ }^{1}$ Their patient, like ours, required systemic corticosteroids and had failed multiple steroid sparing agents and anticoagulants. The response of these two patients suggests that inflammation plays an important role in the pathogenesis of $\mathrm{LV}$, at least in some patients. The purpose of this review is to briefly trace the history of rituximab in clinical use, explain the rationale for
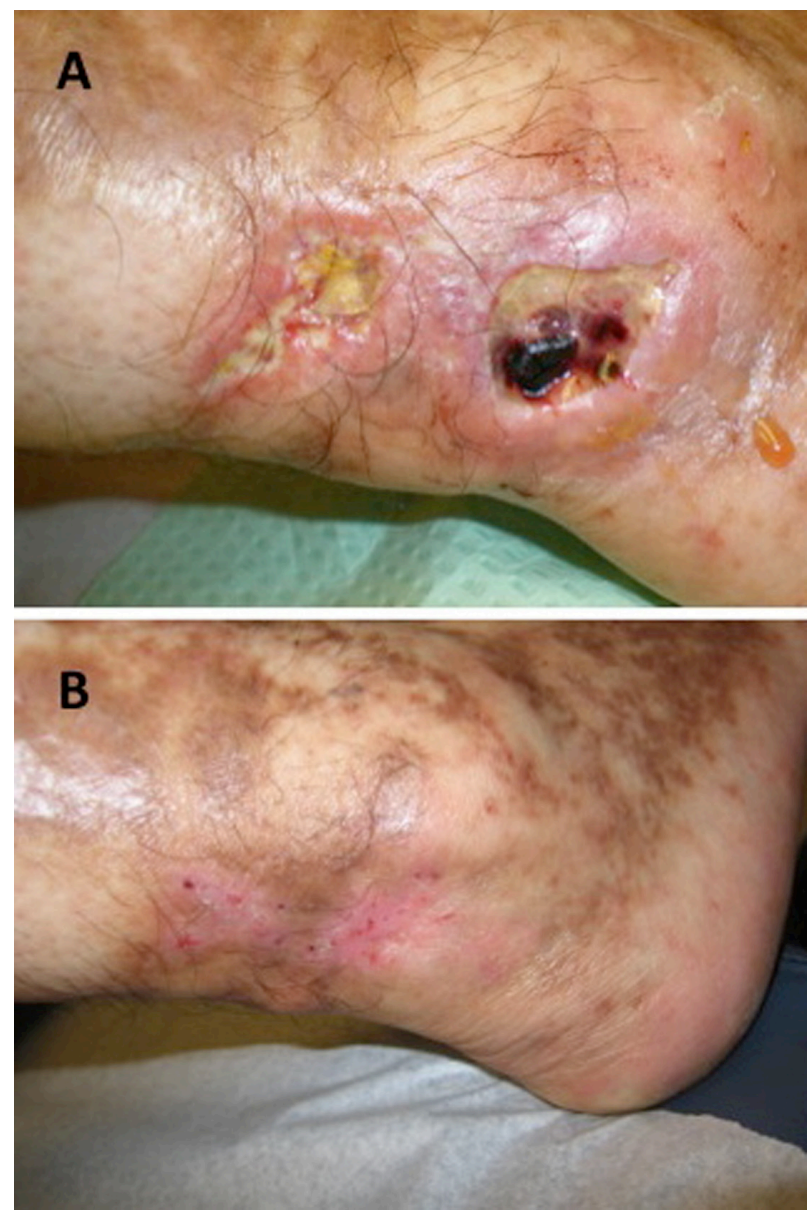

Figure I Livedoid vasculopathy successfully treated with rituximab. A 49-year-old man with reclaitrant ulcers of the lower extremities before $(\mathbf{A})$ and 3-months after (B) treatment with $1000 \mathrm{mg}$ of rituximab infused twice 14 days apart.

this agent in the treatment of auto-inflammatory skin disease, and to discuss dosing in novel disease indications.

\section{The story of rituximab}

Rituximab is a chimeric monoclonal immunoglobulin (Ig) IgG antibody targeted against the CD20 epitope on mature normal and malignant B-lymphocytes. The IgG1 and $\kappa$ constant regions are human, while the variable regions are murine. Rituximab was US Food and Drag Administration (FDA) approved for treatment of follicular non-Hodgkin lymphoma in 1997 on the basis of encouraging phase II and III data reporting a durable response rate of $46 \%$ in heavily pretreated patients with less toxicity than cytotoxic chemotherapy. ${ }^{6}$

Rituximab was the first monoclonal antibody to enter routine use in clinical oncology and has since been demonstrated to improve survival in both indolent and aggressive histology non-Hodgkin lymphoma. ${ }^{7,8}$ 


\section{Mechanism of action}

In many ways, determining the mechanism of action of rituximab in lymphoma was straightforward. Rituximab binding was demonstrated on interim lymph node biopsies in responding patients. Antibody-dependent cell-mediated cytotoxicity and direct apoptosis of malignant B-cells was implicated. The concern with the initial clinical use of rituximab was that a complete knockout of normal peripheral B-lymphocytes could occur. Indeed it did occur in the first trial. Complete absence of peripheral blood B-lymphocytes occurred within one week of initial therapy and persisted until six months when gradual recovery ensued. Surprisingly, there was no significant increase in infections compared to the baseline experienced by heavily pretreated lymphoma patients.

Rituximab did not lead to decreases in immunoglobulin or complement levels either. Although one patient developed detectable human anti-chimeric antibodies these were not quantifiable and did not have any clinical significance. Rituximab levels in serum increased with subsequent treatment. A subset of nine patients had detailed pharmacokinetic sampling with a mean maximum concentration of $500 \pm 135.2 \mu \mathrm{g} / \mathrm{mL}$ (range, 201.4 to $663 \mu \mathrm{g} / \mathrm{mL}$ ) and serum antibody half-life ( $\left.t \frac{1}{2} / 2\right)$ values from 12.7 to 370.8 hours (mean $\mathrm{t}^{1} / 2$ of $225.9 \pm 102.7$ hours). ${ }^{6}$ Responding patients had higher rituximab levels prior to second infusion.

The adverse events associated with the first reports of rituximab were infusion related and tended to be manageable with outpatient care. In addition, these side effects lessened with subsequent administrations during a four-week course of $375 \mathrm{mg} / \mathrm{m}^{2}$ per treatment. Rituximab is routinely prescribed for all non-Hodgkin lymphoma bearing the CD 20 antigen. It has been one of two drugs in hematologic malignancy (imatinib mesylate being the other) that has dramatically changed the treatment landscape in the last fifteen years.

\section{Rituximab in autoimmune disease}

Rheumatoid arthritis (RA) is a classic multisystem autoimmune disease that affects $1 \%$ of the world's population. Women are three to five times more often affected than men, most commonly in the middle-elderly age range (40-70). Rheumatoid factor (present in $80 \%$ of individuals with RA) is an IgM autoantibody to the Fc portion of autologous IgG. Rheumatoid factor complexes with its target and can be found in serum, synovial fluid and synovial membranes. It is diagnostically important, although it is not believed to be causative for the disease. ${ }^{9}$
In fact, RA has classically been conceptualized as a T-cell mediated disease. Past and modern therapeutic efforts in RA have focused on cell mediated immunity or cytokines (particularly tumor necrosis factor) produced by T-cells. That understanding of RA pathophysiology was altered by reports of successful treatment with rituximab. Edwards and colleagues described the treatment of 161 patients with severe RA with methotrexate alone versus one of three regimens containing rituximab $1000 \mathrm{mg}$ fixed dose on days 1 and $15 .{ }^{10}$ Significant clinical improvement in disease symptoms was realized at 24 and 48 weeks in rituximab treated patients. Adverse events mirrored past clinical experience with non-Hodgkin lymphoma and tended to involve mild-moderate infusion toxicity.

A subsequent pharmacokinetic study of this trial described a basic two-compartment model with linear elimination pharmacokinetics. ${ }^{11}$ Typical clearance (CL) was $276 \mathrm{~mL} /$ day and typical central volume of distribution (Vc) of rituximab was $2980 \mathrm{~mL}$ or $45 \mathrm{mg} / \mathrm{kg}$, which is equal to human plasma volume. These investigators also demonstrated an increasing $\mathrm{t}^{1} / 2$ of rituximab with subsequent infusions. The distribution half-life was 2.4 days while the elimination half-life was 19.7 days. The first phase represented elimination of rituximab and distribution from the intravascular to extravascular space. During the second (terminal) phase antibody concentrations slowly declined predominately due to elimination. Covariate analysis to uncover causes of interindividual variability revealed body surface area (BSA) and gender as most significant. However, BSA was only responsible for $19.7 \%$ of interindividual variability suggesting that although BSA predicted CL, the effect may not be highly contributory. These authors interpreted the data to support fixed dosing of rituximab for rheumatoid arthritis. Intravenous (IV) administration of two doses of $1000 \mathrm{mg}$ given two weeks apart is the FDA-approved dose for RA and is widely used in clinical practice.

\section{Rituximab in autoimmune skin disorders}

Pemphigus disorders represent the paradigm of immunobullous skin disease. The pemphigus diseases include pemphigus vulgaris (PV), pemphigus foliaceus (PF) and paraneoplastic pemphigus (PNP), all of which are associated with antibodies directed towards cell-cell adhesion molecules. The pathogenesis of both PV and PF has been well characterized and is dependent on the development of pathogenic anti-desmoglein antibodies, which disrupt keratinocyte-keratinocyte adhesion leading to the clinical findings of blisters and erosions 


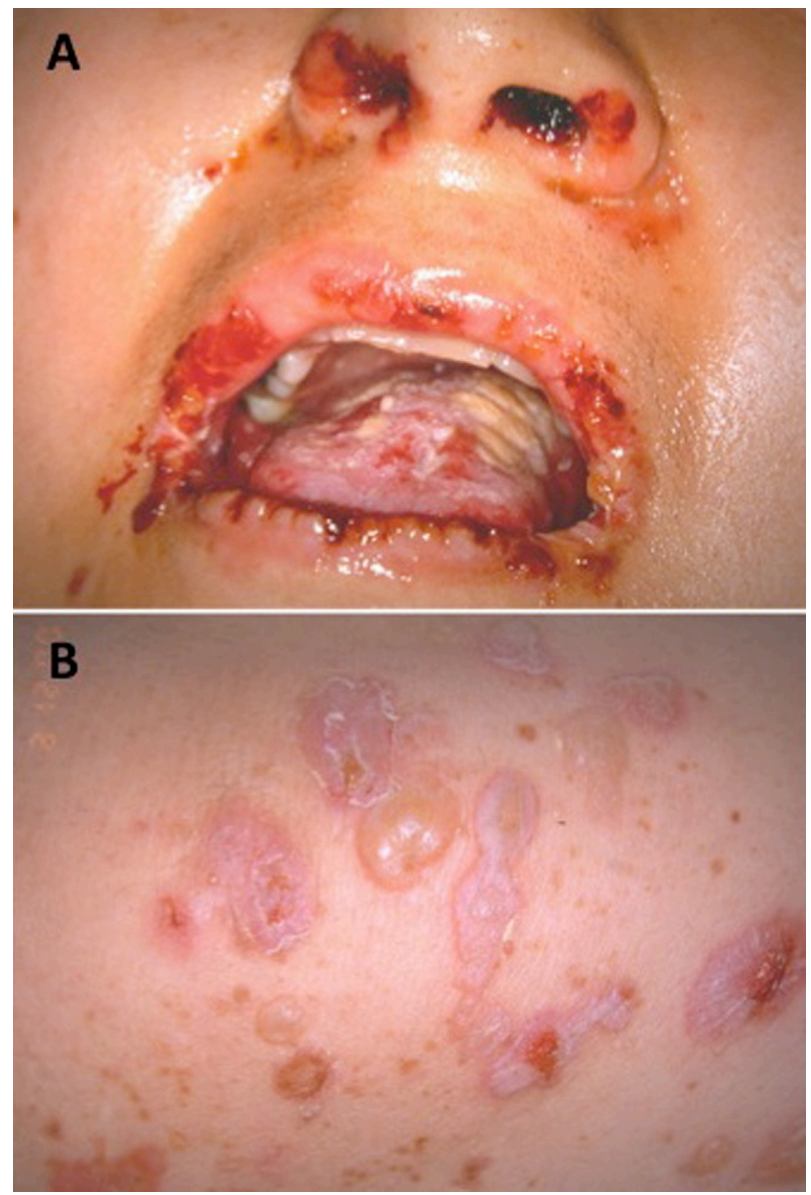

Figure 2 Pemphigus vulgaris. A patient demonstrating mucosal erosions (A) and cutaneous blistering (B).(Image courtesy of Dr Ronald Grimwood).

(Figure 2). PF does not involve mucous membranes and is associated with autoantibodies targeting desmoglein-1. $\mathrm{PV}$, in contrast, is associated with pathogenic antibodies targeting desmoglein-3, which is required for keratinocytekeratinocyte adhesion in mucosal epithelium. Patients with PV also demonstrate dsg-1 autoantibodies that, in combination with dsg-3 autoantibodies, produce epidermal bullae. As an antibody-mediated disorder, pemphigus has been an attractive target for B-cell directed therapies, including rituximab. ${ }^{12}$

Of all the skin diseases reported to have been successfully treated with rituximab, PV has been the most frequently described and the best studied. PV is a life-threatening illness that requires aggressive and long-term therapy with corticosteroids and immunosuppressive medications. While the disease itself is fatal if left untreated, the treatment of the disease is associated with significant morbidity. Some patients also demonstrate a treatment-resistant disease. El Tal and colleagues reviewed 11 reports of the use of rituximab to treat PV between 2002 and 2005 and found that of 17 patients treated, 9 were free of disease for at least six months. ${ }^{13}$ Only one patient did not respond, and one died of an infectious complication five months after treatment. Most patients received rituximab $375 \mathrm{mg} / \mathrm{m}^{2}$ weekly for 4 consecutive weeks. Their review suggested that not only could rituximab successfully treat PV but also that a single cycle could induce long-term remission.

Subsequent reports have demonstrated the efficacy of rituximab in the treatment of PV. Ahmed and colleagues treated 11 patients with rituximab combined with intravenous immune globulin producing long-term remissions in nine patients, all of whom had rapid and sustained loss of desmoglein antibodies coincident with the clinical resolution and tapering of corticosteroids. ${ }^{14}$ Joly and colleagues similarly showed the dramatic response to rituximab alone (375 mg $/ \mathrm{m}^{2} \times 4$ weekly infusions) in steroid-refractory PV and PF. ${ }^{15}$ In their study, 18 of 21 patients obtained complete remission after three months and most demonstrated a stark reduction in desmoglein autoantibodies. In both studies, responses tended to correlate with the reduction in autoantibodies. Mouquet and colleagues later reported a thorough study of the immunologic effects of rituximab treatment on the patients reported by Joly et al. ${ }^{15,16}$ They demonstrated, as expected, a rapid depletion of peripheral B-cells and a drop in IgM after therapy with rituximab. They also demonstrated that while anti-desmoglein antibodies dropped, antibodies directed towards pathogens did not, and B-cell reconstitution was not necessarily associated with the redevelopment of pathogenic autoantibodies. Also of interest, was their observation that some "complete responders" maintained detectable levels of desmoglein autoantibodies, suggesting that other, non-antibody-mediated mechanisms were at play in the pathogenesis of the disease. The obvious corollary is that rituximab must have immunomodulatory effects beyond the simple depletion of pathogenic antibodies. In fact, Eming and colleagues treated $11 \mathrm{PV}$ patients and showed that rituximab induced the expected clinical improvement associated with B-cell and autoantibody depletion and also indirectly decreased the frequency of autoreactive $\mathrm{CD} 4^{+}$T-cells. ${ }^{17}$ The authors presume that B-cells act as antigen presenting cells in the pathogenesis of the disease.

PNP is, as the name implies, a paraneoplastic syndrome with a diverse clinical presentation. The disease virtually always presents with severe stomatitis, although other cutaneous findings vary from lichenoid papules and plaques to frank blistering and erosions. Palms, soles and paronychial tissues may also be affected. The disease has been associated with many malignancies, although non-Hodgkin's 
lymphoma, chronic lymphocytic leukemia (CLL) and Castleman's disease are most frequently reported. ${ }^{18}$ Other organ systems, usually the lungs, are frequently involved, leading to the suggestion that the name paraneoplastic autoimmune multi-organ syndrome is more descriptive. ${ }^{19}$ The pathogenesis of the disease is quite complex. Autoantibodies to many adhesion molecules have been described, and cytotoxic T-cells have also been found to play an important role. ${ }^{20}$ Case reports have demonstrated both success and failure in the treatment of PNP with rituximab. ${ }^{21}$ Two authors of this review (DB and CC) have encountered a patient with progressive PNP despite prior treatment of CLL with rituximab. 22

\section{Other immunobullous disorders Bullous pemphigoid and epidermolysis bullosa acquisita}

Bullous pemphigoid (BP) and epidermolysis bullosa acquisita (EBA) are blistering disorders associated with autoantibodies to bullous pemphigoid antigens (BPAG-1 and BPAG 2) and type VII collagen, respectively. The successful treatment of bullous pemphigoid with rituximab has been reported in both adults and in a child. ${ }^{23,24}$ At least two case reports describe the successful treatment of refractory EBA in adults with rituximab. ${ }^{25,26}$ All of the adults were treated with rituximab $375 \mathrm{mg} / \mathrm{m}^{2}$ for four weeks.

Schmidt and colleagues reported additional cases of immunobullous disease treated with rituximab: two BP, one adult and one child; four PV; and one mucous membrane pemphigoid (MMP).${ }^{27}$ Of note, three of these patients developed severe side effects, including one patient with fatal pneumonia. Mucous membrane pemphigoid is an immunobullous disorder associated with autoantibodies targeting epidermal-dermal basement membrane antigens. The treatment of MMP with rituximab has been described in at least one additional patient. ${ }^{28}$

\section{Atopic dermatitis}

Atopic dermatitis (AD) is a chronic skin disorder manifested by severe itching, a chronic course, and an association with skin barrier defects. The disease is associated with food allergy, asthma, and allergic rhinitis, and most patients with the disease develop elevated IgE levels. The mainstay of treatment involves topical therapies, both anti-inflammatory and moisturizing, although many patients require systemic medications, including corticosteroids, cyclosporine and mycophenolate. ${ }^{29}$ Simon and colleagues reported the successful treatment of severe AD with rituximab (1000 mg IV two weeks apart) in six patients. ${ }^{30}$ All patients demonstrated significant clinical and histological improvement despite only a 50\% reduction in cutaneous B-cells. Allergen-specific IgE levels were also unchanged. This suggests that the behavior of B-cells as antigen presenting cells and activators of T-cells plays an important role in the pathogenesis of AD.

Sediva and colleagues subsequently described two severe $\mathrm{AD}$ cases that failed to respond significantly to rituximab treatment. ${ }^{31}$ This observation may be explained by the lower dose of rituximab (500 mg two weeks apart) used and by the fact that their patients had a particularly severe form of the disease. It also highlights the importance of overcoming reporting bias to share negative results.

\section{Other inflammatory skin diseases}

The successful use of rituximab in the treatment of multiple other skin disorders has been reported. These include dermatomyositis, systemic sclerosis (scleroderma), lupus, and multiple forms of vasculitis. These disorders are of poorly defined pathogenesis, affect multiple organs systems, and are frequently encountered or managed by dermatologists due to severe skin manifestations. The literature supporting the use of rituximab for these disorders varies from isolated case reports to small controlled trials.

A report of three cases of dermatomyositis treated with rituximab $\left(375 \mathrm{mg} / \mathrm{m}^{2} \times 4\right.$ doses $)$ demonstrated significant improvement in skin disease. ${ }^{32}$ However, a pilot trial involving eight patients using $1 \mathrm{~g}$ infused 14 days apart failed to show similar improvement. ${ }^{33}$ Others report success with rituximab in the treatment of both skin and muscle disease. ${ }^{34}$ The use of rituximab in the treatment of systemic sclerosis has recently been reviewed. ${ }^{35}$ The authors review four uncontrolled trials, one open-label randomized controlled trial, and one case report and found inconsistent improvement in skin thickening and interstitial lung disease. Further trials for both diseases are warranted.

The primary vasculitis disorders are a heterogeneous group of inflammatory autoimmune disorders unified by necrotizing destruction of blood vessels. While some are benign and self-limited, others are chronic, recalcitrant to treatment, and associated with significant morbidity and mortality. The nature of the clinical disease varies depending on the organ systems involved and the chronicity of the disease. Multiple authors report the successful use of rituximab in various vasculitic disorders, including Henoch-Shoenlein purpura, cryoglobulinemic vasculitis, urticarial vasculitis, and antineutrophil cytoplasmic antibody-associated vasculitis. ${ }^{36-39}$ Interestingly, vascu- 
litis has also been reported as a side effect of rituximab therapy. ${ }^{41}$

Cutaneous lupus erythematosus may occur in patients with or without a diagnosis of systemic lupus erythematosus (SLE). Risselada and Kallenberg report two patients with SLE whose severe skin manifestations responded to rituximab. ${ }^{41}$ Uthman and colleagues reported the improvement of severe subacute cutaneous lupus erythematosus associated with SLE after treatment with rituximab. ${ }^{42}$ Rituximab may be considered in patients with severe lupus skin disease that is recalcitrant to topical medications, antimalarials, and more conventional immunosuppressive medications.

\section{New directions}

The use of rituximab in severe autoimmune skin disorders has provided a welcome clinical option. Dosing has mirrored either the experience with lymphoid malignancy $\left(375 \mathrm{mg} / \mathrm{m}^{2} \times 4\right.$ weeks $)$ or autoimmune disease $(1000 \mathrm{mg} \times 2$ given two weeks apart). Both dosing schema lead to an increased $t^{1} / 2$ of rituximab on subsequent infusion. In our introductory case we emphasized that the pathophysiology of LV is controversial. Some emphasize an inflammatory mechanism while others emphasize hypercoagulability. In reality, inflammation and procoagulant states are not easily separated. When a disease such as RA or pemphigus vulgaris, previously treated with therapies directed toward cellular immunity, responds to rituximab then our fundamental understanding of pathophysiology is altered as well. T-cells and B-cells interact, and cognitive separation of the two systems is conceptually necessary although likely artificial. Our hope is that this brief review will help to place the use of rituximab in severe skin disorders into historical and scientific context and that we will encourage others to report findings, both positive and negative in the treatment of rare diseases such as LV.

\section{Acknowledgments}

The authors gratefully acknowledge assistance in manuscript preparation by Ms Anjie Colvin, Executive Assistant at Scott \& White Healthcare, Temple, TX USA. The authors also wish to thank our patient with LV who was enthusiastic in sharing his story to further knowledge concerning his rare disease.

\section{Disclosure}

The authors report no conflicts of interest relevant to this research.

\section{References}

1. Zeni P, Finger E, Scheinberg MA. Successful use of rituximab in a patient with recalcitrant livedoid vasculopathy. Ann Rheum Dis. 2008;67(7):1055-1056.

2. Hairston BR, Davis MDP, Pittelkow MR, Ahmed I. Livedoid vasculopathy: Further evidence for procoagulant pathogenesis. Arch Dermatol. 2006;142(11):1413-1418.

3. Toth C, Trotter M, Clark A, Zochodne D. Mononeuropathy multiplex in association with livedoid vasculitis. Muscle Nerve. 2003;28(5): 634-639.

4. Khenifer A, Thomas L, Balme B, Dalle S. Livedoid vasculopathy: thrombotic or inflammatory disease? Clin Exp Dermatol. 2009; Epub 2009 Oct 23.

5. Ishibashi M, Miyamoto J, Nagasaka T, Chen K. Livedoid vasculopathy with underlying subcutaneous necrotizing venulitis in an asymptomatic hepatitis b virus carrier: is livedoid vasculopathy a true nonvasculitic disorder? Am J Dermatopathol. 2009;31(3):293-296.

6. Maloney D, Grillo-Lopez A, White C, et al. IDEC-C2B8 (Rituximab) anti-CD20 monoclonal antibody therapy in patients with relapsed lowgrade non-Hodgkin's lymphoma. Blood. 1997;90(6):2188-2195.

7. Coiffier B, Lepage E, Briere J, et al. CHOP chemotherapy plus rituximab compared with CHOP alone in elderly patients with diffuse large-B-cell lymphoma. N Engl J Med. 2002;346(4):235-242.

8. Schulz H, Bohlius J, Skoetz N, et al. Chemotherapy plus Rituximab versus chemotherapy alone for B-cell non-Hodgkin's lymphoma. Cochrane Database Syst Rev. 2007;(4).

9. Kumar V, Abbas A, Fausto N, Aster J. Robbins and Cotran Pathologic Basis of Disease, 8th ed. Philadelphia, PA: Saunders Elsevier; 2010.

10. Edwards J, Szczepariski L, Szechiriski J, et al. Efficacy of B-celltargeted therapy with rituximab in patients with rheumatoid arthritis. N Engl J Med. 2004;350(25):2572-2581.

11. Ng CM, Bruno R, Combs D, Davies B. Population pharmacokinetics of rituximab (anti-CD20 monoclonal antibody) in rheumatoid arthritis patients during a phase II clinical trial. J Clin Pharmacol. 2005;45:792-801.

12. Edgin WA, Pratt TC, Grimwood R. Pemphigus Vulgaris and Paraneoplastic Pemphigus. Oral Maxillofacial Surg Clin N Am. 2008;20(4):577-584.

13. El Tal AK, Posner MR, Spigelman Z, Ahmed AR. Rituximab: A monoclonal antibody to CD20 used in the treatment of pemphigus vulgaris. $J$ Am Acad Dermatol. 2006;55(3):449-459.

14. Ahmed AR, Spigelman Z, Cavacini LA, Posner M. Treatment of pemphigus vulgaris with rituximab and intravenous immune globulin. N Engl J Med. 2006;355(17):1772-1779.

15. Joly P, Mouquet H, Roujeau J-C, et al. A Single cycle of rituximab for the treatment of severe pemphigus. $N$ Engl J Med. 2007;357(6):545-552.

16. Mouquet H, Musette P, Gougeon M-L, et al. B-cell depletion immunotherapy in pemphigus: effects on cellular and humoral immune responses. J Invest Dermatol. 2008;128(12):2859-2869.

17. Eming R, Nagel A, Wolff-Franke S, Podstawa E, Debus D, Hertl M. Rituximab exerts a dual effect in pemphigus vulgaris. J Invest Dermatol. 2008;128(12):2850-2858.

18. Anhalt GJ. Paraneoplastic pemphigus. J Investig Dermatol Symp Proc. 2004;9(1):29-33.

19. Nguyen VT, Ndoye A, Bassler KD, et al. Classification, clinical manifestations, and immunopathological mechanisms of the epithelial variant of paraneoplastic autoimmune multiorgan syndrome. Arch Dermatol. 2001;137(2):193-206.

20. Billet SE, Grando SA, Pittelkow MR. Paraneoplastic autoimmune multiorgan syndrome: Review of the literature and support for a cytotoxic role in pathogenesis. Autoimmunity. 2006;36(7):617-630.

21. Hoque SR, Black MM, Cliff S. Paraneoplastic pemphigus associated with CD20-positive follicular non-Hodgkin's lymphoma treated with rituximab: a third case resistant to rituximab therapy. Clin Exp Dermatol. 2007;32(2):172-175.

22. Bennett DD, Busick TL. Delayed detection of autoantibodies in paraneoplastic pemphigus. J Am Acad Dermatol. 2007;57(6):1094-1095. 
23. Saouli Z, Papadopoulos A, Kaiafa G, Girtovitis F, Kontoninas Z. A new approach on bullous pemphigoid therapy. Ann Oncol. 2008; 19(4):825-826.

24. Schulze J, Bader P, Henke U, Rose MA, Zielen S. Severe bullous pemphigoid in an infant - successful treatment with rituximab. Pediatr Dermatol. 2008;25(4):462-465.

25. Crichlow SM, Mortimer NJ, Harman KE. A successful therapeutic trial of rituximab in the treatment of a patient with recalcitrant high-titre epidermolysis bullosa acquisita. $\mathrm{Br} J$ Dermatol. 2007;156(1):194-196.

26. Schmidt E, Benoit S, Brocker E-B, Zillikens D, Goebeler M. Successful adjuvant treatment of recalcitrant epidermolysis bullousa acquisita with anti-cd20 antibody rituximab. Arch Dermatol. 2006;142(2):147-150.

27. Schmidt E, Seitz CS, Benoit S, Brocker EB, Goebeler M. Rituximab in autoimmune bullous diseases: mixed responses and adverse effects. Br J Dermatol. 2007;156(2):352-356.

28. Taverna JA, Lerner A, Bhawan J, Demierre M-F. Successful adjuvant treatment of recalcitrant mucous membrane pemphigoid with anti-cd20 antibody rituximab. J Drugs Dermatol. 2007;6(7):731-732.

29. Cork MJ, Danby SG, Vasilopoulos Y, et al. Epidermal barrier dysfunction in atopic dermatitis. J Invest Dermatol. 2009;129(8):1892-1908.

30. Simon D, Hosli S, Kostylia G, Yawalkar N, Simon H. Anti-CD20 (rituximab) treatment improves atopic eczema. J Allergy Clin Immunol. 2008;121(1):122-128.

31. Sediva A, Kayserova J, Vernerova E, et al. Anti-CD20 (rituximab) treatment for atopic eczema. J Allergy Clin Immunol. 2008;121(6):1515-1516.

32. Dinh HV, McCormack C, Hall S, Prince HM. Rituximab for the treatment of the skin manifestations of dermatomyositis: A report of 3 cases. J Am Acad Dermatol. 2007;56(1):148-153.

33. Chung L, Genovese MC, Fiorentino DF. A pilot trial of rituximab in the treatment of patients with dermatomyositis. Arch Dermatol. 2007;143(6):763-767.
34. Rios-Fernandez R, Callejas Rubio JL, Sanchez Cao D, Saez Moreno JA, Orteno Centno N. Rituximab in the treatment of dermatomyositis and other inflammatory myopathies. A report of 4 cases and review of the literature. Clin Exp Rheumatol. 2009;27(6):1009-1016.

35. Daoussis D, Liossis SNC, Tsamandas AC, et al. Is there a role for b-cell depletion as therapy for scleroderma? A case report and review of the literature. Semin Arthritis Rheum. 2009; Epub 2009 Dec 11.

36. Donnithome KJ, Atkinson $\mathrm{P}$, Hinze $\mathrm{CH}$, et al. Rituximab therapy for severe refractory chronic Henoch-Schoenlein purpura. $J$ Pediatr. 2009;155(1):136-139.

37. Tallarita T, Galiano M, Corona D, et al. Successful combination of Rituximab and plasma exchange in the treatment of cryglobulinemic vasculitis with skin ulcers: a case report. Cases J. 2009;2:7859.

38. Mukhtyar C, Misbah S, Wilkinson J, Wordsworth P. Refractory urticarial vasculitis responsive to anti-B-cell therapy. $\mathrm{Br} J$ Dermatol. 2009; 160(2):470-472.

39. Jones RB, Ferraro AJ, Chaudhry AN, et al. A multicenter survey of rituximab therapy for refractory antineutrophil cytoplasmic antibodyassociated vasculitis. Arthritis Rheum. 2009;60(7):2156-2168.

40. Kim MJ, Kim HO, Kim HY, Park YM. Rituximab-induced vasculitis: A case report and review of the medical published work. J Dermatol. 2009;36(5):284-287.

41. Risselada AP, Kallenberg CG. Therapy-resistant lupus skin disease successfully treated with rituximab. Rheumatology. 2006;45(7): 915-916.

42. Uthman I, Taher A, Abbas O, Menassa J, Ghosn S. Successful treatment of refractory skin manifestations of systemic lupus erythematosus with rituximab: Report of a case. Dermatology. 2008;216(3):257-259.
Clinical Pharmacology: Advances and Applications

\section{Publish your work in this journal}

Clinical Pharmacology: Advances and Applications is an international, peer-reviewed, open access journal publishing original research, reports, reviews and commentaries on all areas of drug experience in humans. The manuscript management system is completely online and includes a very quick and fair peer-review system, which is all easy to use.

\section{Dovepress}

Visit http://www.dovepress.com/testimonials.php to read real quotes from published authors. 